\title{
Survival of Mammary Stem Cells in Suspension Culture: Implications for Stem Cell Biology and Neoplasia
}

\author{
Gabriela Dontu ${ }^{1,2}$ and Max S. Wicha ${ }^{1}$
}

There is increasing evidence that a variety of neoplasms including breast cancer may result
from transformation of normal stem and progenitor cells. In the past, isolation and character-
ization of mammary stem cells has been limited by the lack of suitable culture systems able to
maintain these cells in an undifferentiated state in vitro. We have recently described a culture
system in which human mammary stem and progenitor cells are able to survive in suspension
and produce spherical colonies composed of both stem and progenitor cells. Recent obser-
vation that adult stem cells from other tissues may also retain the capacity for growth under
anchorage independent conditions suggests a common underlying mechanism. We propose
that this mechanism involves the interaction between the canonical Wnt signal pathway and
E-cadherin. The Wnt pathway has been implicated in normal stem cell self-renewal in vivo.
Furthermore, there is evidence that deregulation of this pathway in the mammary gland and
other organs may play a key role in carcinogenesis. Thus, the development of in vitro suspen-
sion culture systems not only provides an important new tool for the study of mammary cell
biology, but also may have important implications for understanding key molecular pathways
in both normal and neoplastic stem cells.

KEY WORDS: stem cells; cancer stem cells; breast cancer; mammary gland development; Wnt signaling; self-renewal; differentiation.

\section{INTRODUCTION}

The isolation and characterization of tissue specific stem and progenitor cells has gained impetus due to the important insights that their study can provide into normal development and carcinogenesis. Adult stem cells are defined by their capacity for self-renewal and differentiation into cell lineages present in a specific tissue $(1,2)$. Self-renewal ensures the propagation of the stem cell compartment, which in turn sustains morphogenesis, tissue repair and maintenance. Differentiation generates the specialized cells that form each organ and ensure its normal

\footnotetext{
${ }^{1}$ Department of Internal Medicine, Hematology-Oncology, Comprehensive Cancer Center, University of Michigan, Ann Arbor, Michigan.

${ }^{2}$ To whom correspondence should be addressed at 1500 E Medical Center Dr., 7110 CCGC, Ann Arbor, Michigan 48109; e-mail: gdontu@umich.edu.
}

functioning. In many organs, slowly dividing stem cells give rise to a transit, amplifying population of progenitor cells that proliferate actively in response to specific signals. These undifferentiated cells are multipotent and generate the lineage-restricted progenitors that subsequently undergo terminal differentiation (3). Whereas mammary stem cells have not yet been isolated and characterized, their existence in vivo in mice was unequivocally demonstrated by

\footnotetext{
Abbreviations used: ASMA, alpha smooth muscle actin; APC, adenomatosis polyposis coli; BCRP, breast cancer resistance protein; BMI, B-lymphoma MO-MLV insertion region 1; BMP4, bone morhogenetic protein 4; EGF, epidermal growth factor; ESA, epithelial specific antigen; ER, estrogen receptor; FACS, fluorescence-activated cell sorting; FITC, fluorescein isothyocyanate; bFGF, basic fibroblast growth factor; LIF, leukemia inhibitory factor; Muc 1, mucin 1; MMP7, matrix metalloproteinase 7; MTA3, metastasis associated gene 3; NuRD, Nuclear remodeling and deacetylation complex; PR, progesterone receptor; PTEN, phosphatase and tensin homolog; SP, side population.
} 
serial transplantation studies $(4,5)$. Evidence for the existence of adult mammary stem cells and the strategies employed for their isolation and characterization were thoroughly reviewed in several recent papers $(6,7)$. The focus of this review will be on the development of methods for the cultivation of adult mammary stem/progenitor cells and the potential application of these systems in stem cell biology.

\section{IN VITRO PROPAGATION OF NORMAL STEM CELLS IN SUSPENSION CULTURE}

Ideally stem cells are purified by isolation directly from tissue to avoid any artifacts introduced by in vitro manipulation (2). Purification of the stem cells is based on finding a number of morphological and molecular traits, including surface antigens, that distinguish them from all the other cells in the tissue of interest (8). Most stem cell enrichment/purification protocols rely on fluorescenceactivated cell sorting (FACS) or magnetic immunosorting and use sets of antibodies against cell surface proteins. When the molecular signature is not known, which is the case for mammary stem cells, the task of isolating the stem cells involves a tedious process of screening large numbers of putative markers to identify the ones that co-segregate with functional attributes of stemness. As a minimal working definition, these attributes include self-renewal and ability to generate differentiated progeny. In vivo generation of a functional tissue is the ultimate proof of "stemness" of any candidate marker. However, screening large numbers of candidate markers in in vivo transplantation assays in order to find the stem cell signature is impractical. On the other hand, until recently, the lack of a cultivation system that allows for the propagation of mammary progenitors in an undifferentiated state has precluded the testing of self-renewal in vitro.

We have developed a culture system, based on previous work in neural stem cell biology, which overcomes this inability of previous systems to maintain and propagate mammary stem and progenitor cells. In 1992 Reynolds and Weis published a study in which they showed that neural cells isolated from the subventricular zone of rat embryos can proliferate in suspension culture, clonally generating spherical colonies, which they termed neurospheres (9). A clonal analysis of neurospheres demonstrated that $20 \%$ of these cells, proliferating in vitro in response to EGF and bFGF stimulation, were capable of both self-renewal and differentiation along multiple lineages, the defining characteristics of stem cells (10). Two years later the same group showed that neural stem/progenitor cells from adult animals have the same ability to propagate in vitro as neurospheres (11). Since then, numerous studies utilizing cells derived from either the central or peripheral nervous system, from embryonic and adult tissue showed that the ability to generate neurospheres in vitro correlates with the number of stem cells in the tissue of origin (12-14). Implanted into the nervous system of an animal host, neurospheres generate cells that selfrenew as well as differentiate and contribute to the various cellular compartments of the recipient animal's brain $(12,13)$. Therefore, these cells are capable of self-renewal and multilineage differentiation, both in vitro and in vivo. This experimental system has been an extremely useful tool in neural stem cell biology. It was used as an in vitro model for early development $(15,16)$, to identify factors involved in cell fate determination and to unravel signaling pathways active in stem and early progenitor cells (17-19). A series of studies utilized this system for comparative transcriptional profiling in order to identify gene expression changes underlying self renewal or lineage specific differentiation (20-23). Moreover, neurospheres were utilized in a number of in vivo studies to repair neuronal loss in mice with spinal cord and sciatic nerve injuries and in rat models with ischemic cerebral cortex (24), partial Parkinson's disease (25) and Huntington's disease (26). These studies demonstrated successful engraftment, migration to the site of the lesion and differentiation of sphere-derived cells into functional neurons with variable degrees of behavioral and anatomical recovery. These results make the case for the potential use of these progenitor cells, propagated in vitro in suspension culture, in repairing damaged tissue in vivo. The neurosphere culture is also routinely used now to assess the enrichment of stem cells in experiments using cell sorting for identifying markers of stem cells $(12,13)$. Neurosphere formation was also used to assess the size of the stem cell population in neural tissue of genetically manipulated mice in experiments aiming to investigate the role of genes such as BMI (23), PTEN (27), LIF and Notch $(28,29)$ in self-renewal.

\section{NONADHERENT MAMMOSPHERES}

Recently we adopted the suspension culture as a strategy for the in vitro enrichment and propagation 
of human mammary stem/progenitor cells (30). Based on the model of neurospheres, we hypothesized that a small population of mammary cells with stem cell properties would be able to survive and proliferate in the absence of attachment to an exogenous substratum. We developed a culture system in which human mammary epithelial cells, isolated from reduction mammoplasties, are cultured on a non-adhesive substratum in serum-free medium in the presence of EGF and/or bFGF. Under these conditions the vast majority of cells undergo "anoikis." This term specifically applies to the apoptosis of nontransformed cells which occurs in the absence of anchorage to a substratum. We hypothesized that anoikis was a property of differentiated cells, but that stem cells could survive anchorage independent conditions. Consistent with this hypothesis, we found that approximately four in 1000 freshly isolated cells are able to survive and proliferate, and form multicellular spheroids. We termed these spheroids "mammospheres" by virtue of their resemblance to neurospheres cultured from primary neural cells. As is the case for neurospheres, we demonstrated that mammospheres are highly enriched in undifferentiated cells, as demonstrated by the ability of single cells isolated from mammospheres to generate multilineage colonies when cultured in the presence of serum on a collagen substratum which promotes their differentiation (Fig. 1(a)). Primary mammospheres contain eight times more bi-lineage progenitor cells than freshly cultured human mammary cells. Secondary and later passaged mammospheres consist of virtu-

d) Differentiation in vivo
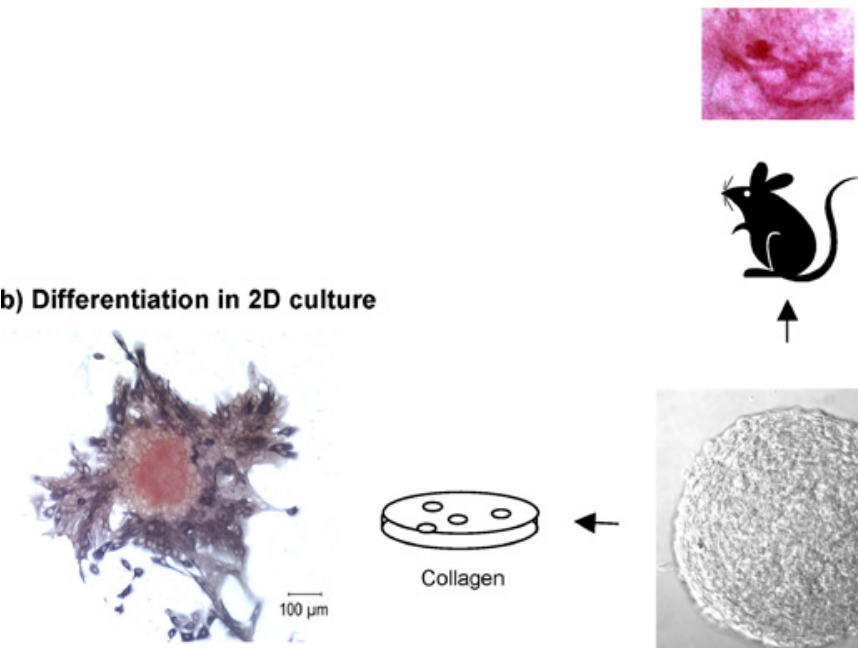

Triple lineage colony

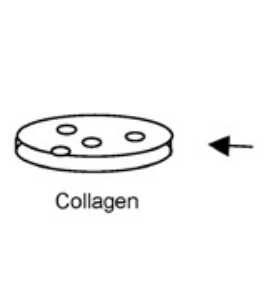

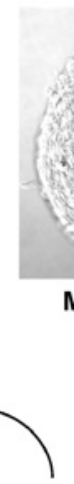
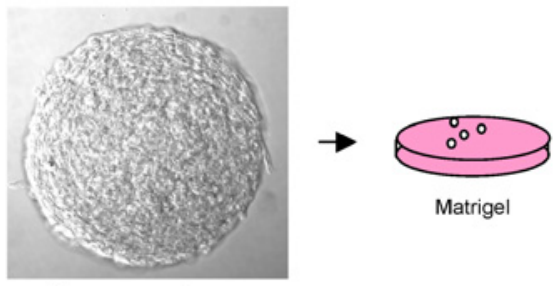

Matrigel

Mammosphere

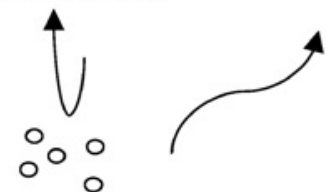

Single cells

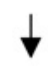

a) Self-renewal

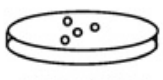

Suspension c) Differentiation in 3D culture

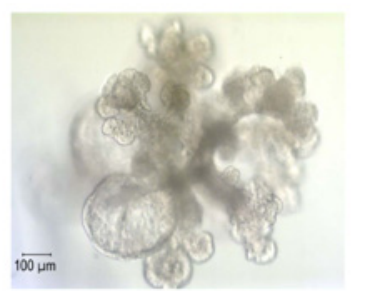

Ductal lobulo-alveolar structure

Fig. 1. Mammospheres are composed of stem/progenitor cells. Mammosphere-derived cells plated at clonogenic densities can: (a) self renew, (b) generate mixed colonies containing cells of all the three mammary lineage types (immunostained with lineage specific markers, ductal epithelial-ESA, brown; myoepithelial-CD10, purple; alveolar, beta-casein—red) (c) generate complex structures in 3D Matrigel culture, (d) generate ductal-alveolar outgrowths in vivo. 
ally $100 \%$ bipotent progenitors. Furthermore, the majority of bipotent progenitors are able to generate colonies that contain all three lineages of the adult mammary gland, myoepithelial, ductal epithelial, and alveolar epithelial cells. We have also shown that mammospheres contain cells capable of clonally generating complex functional structures in reconstituted 3-D culture systems in Matrigel (Figs. 1(c) and 2).

Self-renewal of a cell population within mammospheres was demonstrated utilizing an assay in which single cells from mammospheres are able to generate second and later generation spheres (Fig. 1(b)). We demonstrated that mammospheres were clonally generated using retroviral marking. Furthermore, we demonstrated that mammospheres derived from these passaged cells also have multipotent differentiation potential. These results resemble those reported for neurospheres $(10,31)$ and are consistent with a model in which the mammosphereforming cell represents a mammary stem cell which undergoes limited self renewal and then gives rise to mammary progenitors still capable of multi-lineage differentiation (32). Clonal experiments in which spheres were grown from single cells and single mammospheres were passaged, suggest that one or two self-renewal divisions are involved in the formation of a single mammosphere. This limited number of self-renewal divisions is in agreement with the majority of studies involving adult stem cells, which indicate that expansion of the adult stem population does not readily occur ex vivo, presumably due to asymmetric cell kinetic divisions that result in a large number of progenitors and differentiated cells and a small fixed number of stem cells. Our findings indicate that mammary stem cells are contained in the small population of cells that are anchorage independent and survive suspension culture to proliferate and differentiate into mammary progenitor cells (30).

To determine if the ability of cells to form mammospheres correlates with enrichment in progenitor cells, we tested the sphere formation capability of the side population (SP) of mammary epithelial cells (30). SP represents a subpopulation of cells capable of excluding dyes, such as Rhodamine and Hoechst, due to the expression of transporter proteins, such as BCRP (breast cancer resistance protein) and $P$-glycoproteins (33). It has been shown that the SP fraction of hematopoietic and neural cells contains the long-term repopulating stem cells (34). This phenomenon has also recently been demonstrated for mouse mammary cells with SP properties, which can regenerate the gland upon transplantation (35). In our study SP and non-SP staining population from uncultured cells were separated by FACS and placed in suspension culture. Only cells contained in the SP fraction were capable of mammosphere formation in suspension culture as well as generation of multilineage colonies on collagen substrata (30). In order to assess the cellular composition of mammospheres we used immunostaining with markers specific for the differentiated mammary cells of luminal epithelial (ESA, Muc1, cytokeratin 18) and myoepithelial lineages (CD10, ASMA, cytokeratin 14, alpha 6 integrin). Some of these markers were identified in previous studies as being associated with bi-potent progenitor cells (ESA, alpha 6 integrin) $(36,37)$ or mammary stem/progenitor cells (cytokeratin 5). Mammospheres contained cells positive for alpha 6 integrin, cytokeratin 5 and CD10, ESA and cytokertain 14 (30). ER and PR expression was also detectable by immunostaining in mammospheres and in colonies generated from mammospheres plated on a collagen substratum. A subpopulation of the ER positive cells was also positive for the Ki67 proliferation marker, indicating that these cells can divide in vitro. After 5-7 days of cultivation on a collagen substratum ER expression was no longer detected, suggesting that it is downregulated during differentiation in vitro (unpublished observations).

We have recently found that, when transplanted in the cleared mammary fat pad of NOD/SCID mice, mammospheres generated limited outgrowths with the morphological and cellular characteristics of a human mammary ductal alveolar structure (Figs. 1(d) and 2). As few as 500 transplanted mammospheres $(10,000-25,000$ cells $)$ generated outgrowths, in the absence of human fibroblasts. Improved engraftment was obtained by using mammospheres combined with human mammary fibroblasts, as described by Kuperwasser et al. (38). Titration experiments will be required to determine the absolute enrichment in gland reconstituting activity of mammosphere derived cells.

We have utilized the mammosphere cultivation system to delineate signaling pathways involved in cell fate specification of mammary stem/progenitor cells, such as Notch and Sonic Hedgehog (32). Using assays for in vitro self-renewal and differentiation that we developed with this system, we showed that Notch signaling regulates cell fate decisions in the mammary gland at several distinct developmental stages. Notch activation increases selfrenewal of mammary stem cells, as well as acting on 


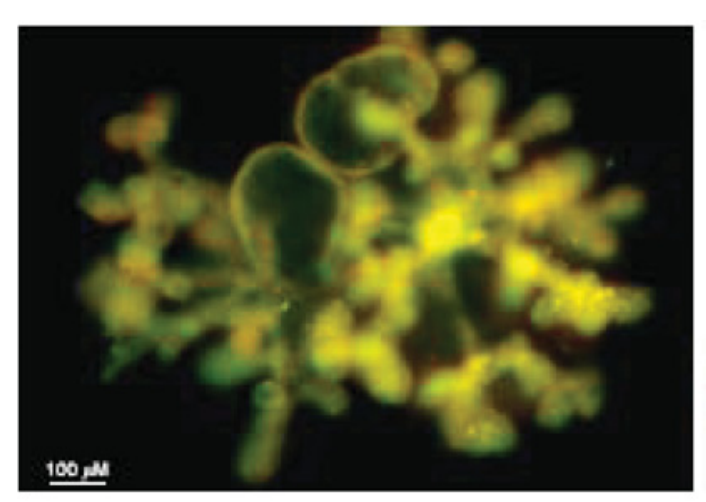

a

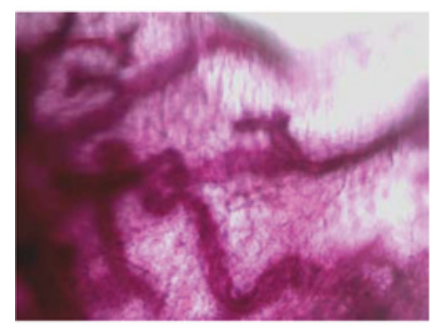

C

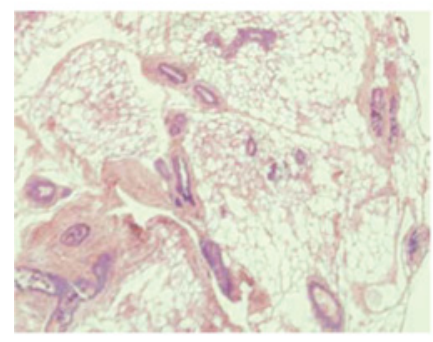

f

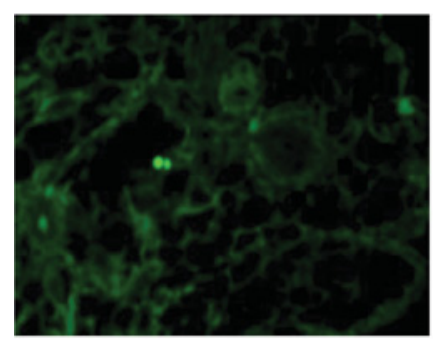

i

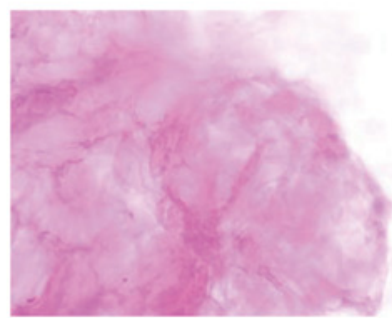

d

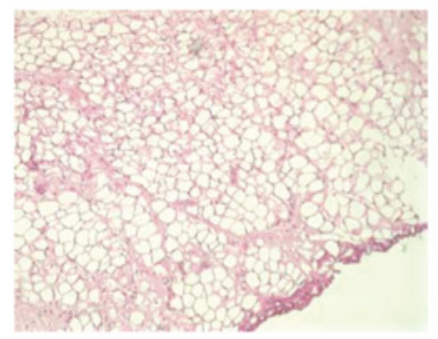

g

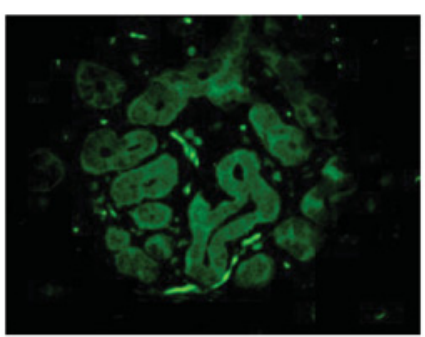

j

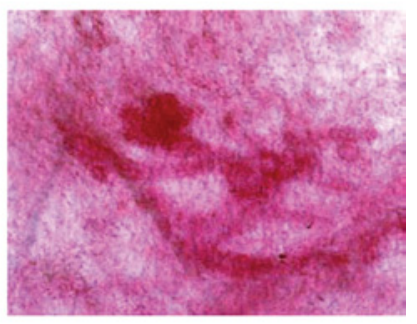

e

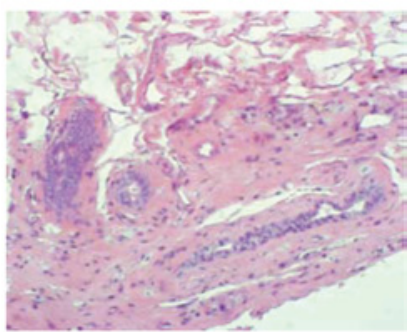

$\mathrm{h}$

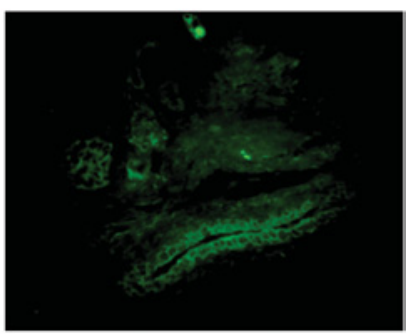

k

Fig. 2. (a) Fully developed ductal-alveolar structure grown in Matrigel from a secondary mammosphere, immunostained with lineage specific markers (myoepithelial cells are stained red with CD10-Texas red and ductal epithelial cells are stained green with ESA-FITC) (b) Same as a, higher magnification. (c-k)Mammospheres generate outgrowths in the cleared mammary fat pad. Whole mount staining (carmine-red) and H\&E tissue sections through a mouse mammary fat pad: not cleared [(c) and (f)], cleared, non-implanted [(d), (g)], cleared and implanted with mammospheres derived from human mammary epithelial cells [(e), (h), (k)]. Immunostaining utilizing human specific ESA antibody - tissue section through a mouse mammary gland (i), human mammary gland (j), mouse cleared fat-pad implanted with human mammospheres $(\mathrm{k})$. 
progenitor cells to promote the adoption of the myoepithelial cell fate at a later step, during differentiation. Sonic hedgehog pathway also appears to have a role in regulating self-renewal of mammary stem cells, probably also interacting with the Notch pathway.

Based on the observations that embryonic stem cells, as well as at least two types of stem/progenitor cells from neural tissue and mammary tissue, have the ability to survive and proliferate in an anchorage independent manner, we speculated that suspension culture might be utilized as a method for isolating adult stem/progenitor cells from other tissues. Indeed this was recently confirmed in the case of skin (39), cardiac (40), and inner ear stem cells (41) that generated the same type of floating spherical colonies in suspension cultures, composed of cells with stem and progenitor functional properties.

\section{CANCER STEM CELLS AND TUMOR SPHERES}

We and others have recently proposed a model in which mammary carcinogenesis is driven by tumor stem cells derived from mutated adult stem or progenitor cells $(7,42)$. This model is based on the widely accepted concept that cancer arises through a series of mutations that may occur over many years. Since adult stem cells are slowly dividing, long-lived cells with a high proliferative capacity, they are able to accumulate the multiple mutations that occur during carcinogenesis $(1,42)$. Being exposed to damaging agents over long periods of time, they can accumulate and propagate the mutations induced by the genotoxic agents. These transformed stem or progenitor cells can in turn become "cancer stem cells," which maintain or acquire functional properties present in normal stem cells, including the capacity for selfrenewal and differentiation. Mutations accumulated in the "cancer stem cell" disrupt the tight control of these stem cell functions, ultimately leading to deregulation of self-renewal, which drives the process of tumorigenesis, and to aberrant differentiation, which generates the cellular heterogeneity found in tumors. The existence of cancer stem cells was first demonstrated in hematologic malignancies and, more recently, in solid tumors (43-46). In a previous review we analyzed in more detail the functional characteristics shared by normal stem cells and cancer cells, such as capacity for self-renewal, ability to differentiate, active telomerase, activation of antiapoptotic pathways, increased membrane transporter activity, anchorage independence and ability to migrate (4). Comparing the transcriptional profile of stem/early progenitor cells with that of more differentiated cells of the mammary epithelium, we proposed that the former more closely resembles that of cancer cells than the latter. This observation suggests an underlying molecular circuitry that makes stem cells more readily transformed than differentiated cells. A key event in transformation may be the deregulation of pathways such as self-renewal, which are already active in stem cells.

Direct evidence for the existence of cancer stem cells was first provided by the work of John Dick's group in hematological malignancies (43). They demonstrated the presence of a cancer stem cell population in human leukemias, representing a very small fraction of the total leukemic population, which was capable of transferring the disease to immunosuppressed mice. Furthermore, leukemic stem cells shared the expression of phenotypic markers with normal hematopoietic stem cells. Based on these studies, they proposed that different leukemic phenotypes resulted from particular mutations in hematopoietic stem cell populations, which resulted in aberrant differentiation of these cells $(43,47)$.

In collaboration with Clarke's group we recently presented evidence for the existence of human breast cancer stem cells (45). Flow cytometry was utilized to separate subpopulations of cells based on their surface marker expression. A subpopulation of tumor cells, with the phenotype CD $44^{+} \mathrm{CD} 24^{-}$ lineage-, which possesses highly tumorigenic characteristics, was thus identified. The tumorigenic subset, representing a minority of the total cellular population within a tumor, was defined by the same markers in the majority of tumors examined. As few as 200 of these cells consistently formed tumors in NOD/SCID mice. In contrast, the bulk of the tumor, which contained cells with different cell surface phenotypes, failed to form tumors even when tens of thousands of cells were injected. In order to determine whether this experimental system merely selected for a highly tumorigenic subset of cells, the phenotype of tumors produced in NOD/SCID mice by the prospectively isolated tumorigenic cells was analyzed. The results showed that the small population of tumorigenic cells was able to regenerate the entire phenotypic heterogeneity found in the initial tumor. These findings support a stem cell model of carcinogenesis, in which a small population of tumorigenic cells, with definable phenotype, is able to give rise to more 
tumorigenic cells as well as the bulk tumor population, without tumorigenic properties. Hence, the tumorigenic stem cells, like their normal counterparts, are able to undergo both self-renewal and differentiation.

The existence of a cancer stem cell phenotype in brain tumors has also been suggested by two groups that utilized in vitro cultivation of brain tumor cells in suspension, as neurospheres $(46,48)$. Singh and coworkers used this experimental system to identify cancer stem cells from various human brain tumors (46). Their study showed a correlation between the self-renewal capacity of cancer stem cells, demonstrated by sphere formation, and the clinical aggressiveness of the brain tumors from which these cells were derived. They also demonstrated that these cancer stem cells from brain tumors had the ability to generate differentiated progeny in vitro, similar to the tumor from which the cells were isolated. Most recently, they have shown that brain cancer stem cells, expressing the neural stem cell marker CD 133 are able to form tumors in NOD/scid mice, whereas CD 133 negative cells are not (47). Moreover, when injected into animals, CD 133+ cells generated heterogeneous tumors composed of both CD 133+ and CD133 - cells. Interestingly, only CD 133+ cells were capable of forming neurospheres.

Thus, as we have previously demonstrated for breast cancer, the stem cell model of carcinogenesis also applies to brain cancers.

\section{HOW DO STEM CELLS AVOID "ANOIKIS"-A THEORETICAL MODEL}

A fundamental question is whether the anchorage independence of normal stem cells is merely an in vitro phenomenon. Does the molecular mechanism responsible for this property also play a role in stem cell behavior in vivo? We will further speculate on this latter possibility and propose a mechanism that might coordinate proliferation and survival during stem/progenitor cell differentiation with cell-cell adhesion and integration into mature tissue architecture. Survival of single cells in suspension culture involves survival in the absence of cell-matrix interaction and cell-cell interactions.

\section{Cell-Matrix Interactions}

Our analysis of mammosphere showed the presence of matrix molecules including tenascin, decorin and laminin (30). Interestingly, decorin and tenascin are present in the embryonic mammary gland, while laminin is also present in the basement membrane of the adult gland, suggesting that mammosphere formation may recapitulate some of the events that occur during embryonic and early mammary development in vivo. It appears that the cells that escape anoikis, represented mainly by stem cells and possibly early progenitor cells, synthesize and deposit extracellular matrix, creating an in vitro niche that supports their survival and proliferation in suspension. Undoubtedly, stromal-epithelial interactions are also involved in the generation of the stem cell developmental niche in vivo. Recent observations by Weinberg's group (38), as well as our own, demonstrate that mammary stroma greatly potentiates the growth and differentiation of human mammary epithelium (from epithelial fragments and mammospheres) in NOD/scid mice. Interactions between the mammosphere-initaiting cells and their progeny and between the cellular and extracellular components of the spheres, dictate the types of divisions (self renewal vs. differentiation) and the cell fate adopted by the cells (lineage commitment). It remains to be determined to what extent this behavior recapitulates events occurring in vivo. One might speculate that signaling initiated by the matrix molecules engages specific sets of integrins expressed by mammary progenitor cells, promoting their survival. Signaling through growth factor receptors present in these cells probably also plays an important role in cell survival. The survival of cancer cells at sites of metastasis may employ similar mechanisms. One important caveat of extrapolating in vitro data to in vivo events has been recently illustrated in the neurosphere system. In vitro conditions altered the potential and fate specification of neural progenitor cells, generating tripotent cells, not seen in vivo (49).

\section{Cell-Cell Interactions}

Cell-cell interactions are crucial for the survival of epithelial cells both in vitro and in vivo. An important component of this process is E-cadherin (50). Loss of this mechanism of adhesion is probably involved in the massive apoptosis that occurs during mammary involution, following lactation (51). Interestingly, the resistance of mammary stem/progenitor cells to apoptosis during involution preserves the populations which regenerate the gland 
during subsequent pregnancies. E-cadherin is a member of a family of single-pass transmembrane glycoproteins that plays a role in establishing cell polarity and tissue morphology (50). The extracellular domain of E-cadherin interacts homotypically with an E-cadherin molecule on an adjacent cell. The cytoplasmic domain of E-cadherin is linked to the cytoskeleton via interaction with catenins. An important interaction is with beta-catenin, a molecule in the canonical Wnt signaling pathway. The connection between E-cadherin and Wnt signaling and its role in normal breast development and carcinogenesis was the subject of an excellent recent review (52). We will discuss this connection from the perspective of carcinogenesis which results from deregulated self-renewal of stem cells or acquired selfrenewal by early progenitor cells. Beta-catenin is present in two cellular locations. In association with E-cadherin, it forms the adherens junctions. In the cytoplasm, beta-catenin is present in a complex with proteins such as adenomatous polyposis coli (APC) and axin. In the absence of Wnt signaling, betacatenin is phosphorylated and targeted for degradation (53). When Wnt is activated, unphosphorylated beta-atenin translocates to the nucleus, where it binds and activates the transcription factors TCFLEF, which then activates a variety of downstream target genes, including c-Myc and cyclin D1 $(54,55)$. It has been recently proposed that the balance between the beta-catenin present in the adherens junctions and that involved in transcriptional activity is controlled by the pool of intracellular APC (56). Furthermore, evidence from Drosophila studies indicates that APC regulates spindle orientation and asymmetric cell division (57). An inverse correlation between E-cadherin and Wnt signalling has been noted in various tissues and organs, during development, as well as in cancers (58). In some human epithelial cancers, E-cadherin gene mutations promote Wnt signalling, as judged by the detection of nuclear beta-catenin in the tumor tissue (58). It has also been shown that E-cadherin can mediate growth suppression by inhibition of beta-catenin signaling, in an adhesion dependent manner (59). In cultured mammary epithelial cells, inhibition of E-cadherindependent cell aggregation predisposes to cell death. In vivo studies, utilizing transgenic mice and the Cre/lox recombinase system, demonstrated that alveolar differentiation during lactation is dependent on the expression of E-cadherin (60). Moreover, as indicated above, disruption of E-cadherin-dependent cell adhesion probably initiates the apoptotic pro- gram during mammary involution. The interaction of E-cadherin with beta-catenin appears to mediate these processes. Furthermore, recent studies provided evidence for a converse mechanism, namely nuclear beta-catenin/Lef1 mediated suppression of E-cadherin expression during normal development of hair follicles in mice (61). The authors propose that the downregulation of E-cadherin may in turn perpetuate the Wnt signalling pathway, by increasing the pool of transcriptionally competent beta-catenin.

The shifting balance between the levels of E-cadherin and cytoplasmic beta-catenin, under the control of Wnt signaling, could remodel cellular junctions, coordinating cell proliferation with cell adhesion during differentiation. We speculate that stem cells and early progenitors, unlike their more differentiated progeny, are not dependent on cell-cell interactions for survival. In support for this hypothesis is the ability of a variety of stem/progenitor cells to survive in suspension culture, as discussed above. Moreover, the mammary small light cells (SLC), described by Smith and Chepko (62), thought to be stem or very early progenitor cells, lack polarity and specialized membrane contacts with neighboring cells. The absence of gap junction proteins, including connexins, in mammary progenitor cells was described by Trosko et al. (63). Utilizing, transcriptional profiling of mammospheres as well as differentiated cells derived from mammospheres, we found that E-cadherin expression level increases threefold during differentiation, while repressors of E-cadherin Snail and Slug are downregulated three- and twofold respectively (30). In the absence of adherens junctions and E-cadherin, most of the beta-catenin will be localized in the cytoplasm. Signaling through the Wnt pathway results in beta-catenin-mediated transcriptional activity that activates different sets of genes, ultimately resulting in self-renewal or differentiation. The outcome probably depends on signals that modulate the Wnt pathway response and the interactions with other pathways involved in fate specification. While differentiation occurs, E-cadherin expression progressively increases, adherens junctions form and the antiapoptotic mechanisms are downregulated. Nuclear beta-catenin and its transcriptional activity decrease (Fig. 3). Consequently, differentiated cells are not able to migrate, depend on cell-cell adhesion for their survival, and proliferate less. In favor of this scenario gene expression changes induced by beta-catenin transactivation show upregulation of c-Myc, Cyclin D1 (proliferation), stromlysin-1, MMP7 and Twist (migration) and 

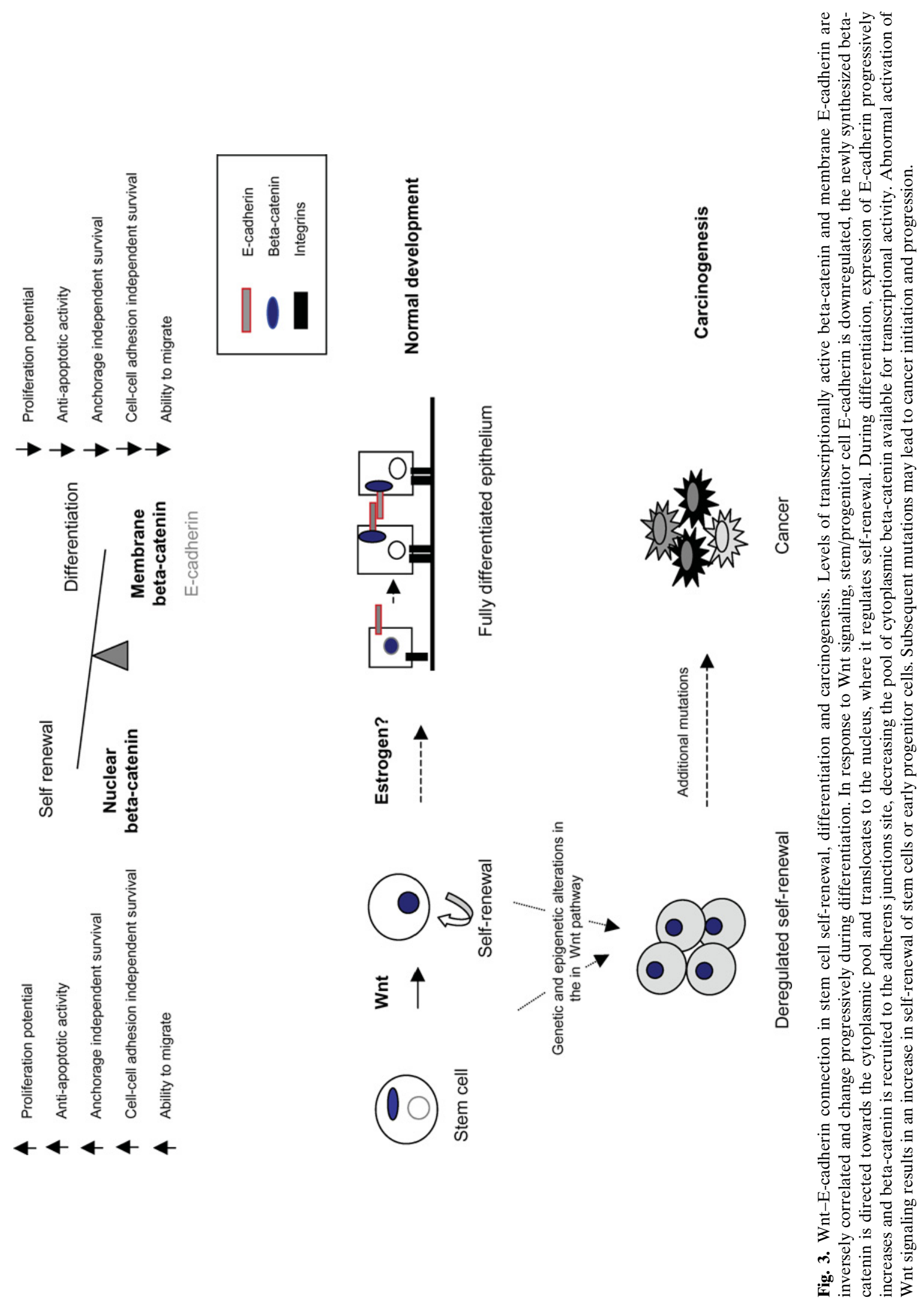
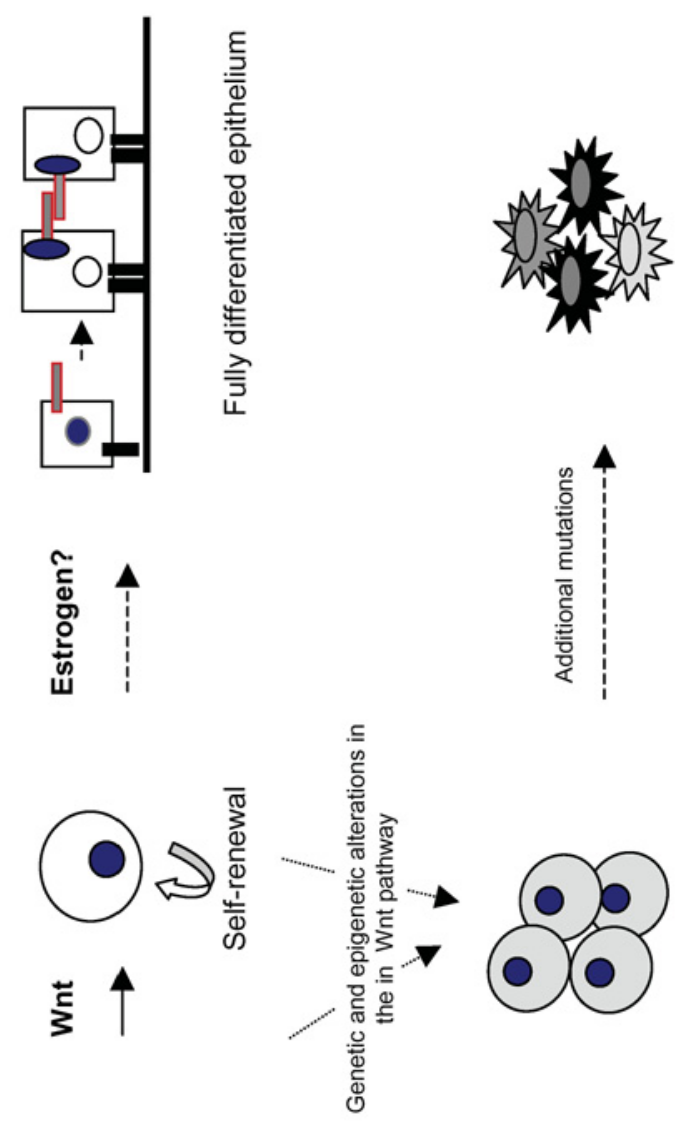

(1)

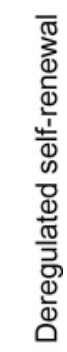


down regulation of E-cadherin, Ephrin, and BMP4. Furthermore, Fujita et al. reported that these events are under hormonal regulation, and involve the estrogen receptor (64). They showed that in the mammary gland E-cadherin expression is regulated by the estrogen receptor through MTA3, a component of the Mi-2/NuRD transcriptional repressor complex which is an ER regulated inhibitor of Snail, a repressor of E-cadherin (64).

During carcinogenesis a key event may be represented by an abnormal activation of Wnt signaling, resulting in an increase in self-renewal of stem cells or early progenitor cells. The Wnt pathway has been associated with both normal development of the mammary gland, from the very early stages of formation of anlage to terminal differentiation during lactation (65-68). Also, increasing evidence points to a link between Wnt signaling and mammary neoplasia (69-71). Moreover, it appears that the role of Wnt activation in self-renewal of stem cells might be the basis of its oncogenic potential (72). A number of studies have recently provided evidence for a direct role of Wnt signaling in the self-renewal of normal hematopoietic, epidermal, and gut stem cells (73-76). A role for Wnt signaling in self-renewal of mammary stem cells was suggested by recent studies of Alexander et al. who utilized transgenic mice to demonstrate that overexpression of Wnt ligands in mammary stroma or activated beta-catenin in mammary epithelium leads to increased numbers of mammary stem cells (77). A direct link between selfrenewal of stem/progenitor cells and carcinogenesis was suggested by the study of Li et al. who demonstrated in a mouse model that expression of the components of Wnt-signaling pathway preferentially induces mammary cancers derived from mammary progenitor cells (70).

Subsequent mutations favored by the increased proliferative activity may occur during cancer initiation and progression. However, some of the traits that confer selective growth advantage to the cancer cells and contribute to local invasion and metastasis are normal attributes of stem cells. The observation that epigenetic mechanisms may be responsible for alterations such as the loss of E-cadherin, during carcinogenesis is consistent with this hypothesis.

\section{CONCLUSIONS}

In this article, we have reviewed the development of suspension-based culture systems for the propagation of stem and progenitor cells from the mammary gland, as well as from other organs. The ability of normal stem cells, as well as their malignant counterparts, to survive in suspension culture suggests a common molecular mechanism that may also have significance for the behavior of stem cells in vivo. We discuss evidence suggesting that the Wnt signal pathway and the E-Cadherin-beta-catenin interaction may play a key role in normal stem cell selfrenewal and differentiation, as well as survival. Furthermore, there is accumulating evidence that deregulation of this pathway plays an important role in a variety of neoplasms, including breast cancer. These neoplasms, in turn, may be driven by a small stem cell component within tumors. This stem cell component, resistant to conventional treatments, may contribute to relapse following therapy. Inhibition of pathways, such as Wnt signaling, may thus prove to be a novel therapeutic strategy for selectively targeting this resistant cell population.

\section{ACKNOWLEDGMENTS}

Thanks are due to Dr Michael Clarke for helpful discussion, Dr Kathleen Ignatosky, Dr Suling Liu, Dr Ilia Mantle, and Dr Nam-shik Ahn for critical review of this paper. This work was supported by NIH Grants Nos. CA66233 and CA101860.

\section{REFERENCES}

(1) Weissman IL. Stem cells: Units of development, units of regeneration, and units in evolution. Cell 2000;100:157-68.

(2) Morrison SJ, Shah NM, Anderson DJ. Regulatory mechanisms in stem cell biology. Cell 1997;88:287-98.

(3) Weissman IL, Anderson DJ, Gage F. Stem and progenitor cells: Origins, phenotypes, lineage commitments, and transdifferentiations. Annu Rev Cell Dev Biol 2001;17:387-403.

(4) Daniel C, Young L, Medina D, DeOme K. The influence of mammogenic hormones on serially transplanted mouse mammary gland. Exp Gerontol 1971;6:95-101.

(5) Kordon EC, Smith GH. An entire functional mammary gland may comprise the progeny from a single cell. Development 1998;125:1921-30.

(6) Smalley M, Ashworth A. Stem Cells and breast cancer: A field in transit. Nat Rev Cancer 2003;3:832-44.

(7) Dontu G, Al-Hajj M, Abdallah W, Clarke M, Wicha MS. Stem cells in normal breast development and breast cancer. Cell Proliferat 2003;36:59-72.

(8) Blau HM, Brazelton TR, Weimann JM. The evolving concept of a stem cell: Entity or function? Cell 2001;105:829-41.

(9) Reynolds B, Tetzlaff W, Weiss S. A multipotent EGFresponsive striatal embryonic progenitor cell produces neurons and astrocytes. J Neurosci 1992;12:4565-74. 
(10) Reynolds BA, Weiss S. Clonal and population analyses demonstrate that an EGF-responsive mammalian embryonic CNS precursor is a stem cell. Dev Biol 1996;175:1-13.

(11) Morshead C, Reynolds B, Craig C, McBurney M, Staines $\mathrm{W}$, Morassutti D, et al. Neural stem cells in the adult mammalian forebrain: A relatively quiescent subpopulation of subependymal cells. Neuron 1994;13:1071-82.

(12) Uchida N, Buck DW, He D, Reitsma MJ, Masek M, Phan $\mathrm{TV}$, et al. Direct isolation of human central nervous system stem cells. Proc Natl Acad Sci 2000;97:14720-5.

(13) Rietze RL, Valcanis H, Brooker GF, Thomas T, Voss AK, Bartlett PF. Purification of a pluripotent neural stem cell from the adult mouse brain. Nature 2001;412:736-9.

(14) Johansson C, Momma S, Clarke D, Risling M, Lendahl U, Frisen J. Identification of a neural stem cell in the adult mammalian central nervous system. Cell 1999;96:25-34.

(15) Kukekov VG, Laywell ED, Suslov O, Davies K, Scheffler B, Thomas LB, et al. Multipotent stem/progenitor cells with similar properties arise from two neurogenic regions of adult human brain. Exp Neurol 1999;156:333-44.

(16) Kukekov VG, Laywell ED, Thomas LB, Steindler DA. A nestin-negative precursor cell from the adult mouse brain gives rise to neurons and glia. Glia 1997;21:399-407.

(17) Caldwell MA, He X, Wilkie N, Pollack S, Marshall G, Wafford KA, et al. Growth factors regulate the survival and fate of cells derived from human neurospheres. Nat Biotechnol 2001;19:475-9.

(18) Zhu G, Mehler MF, Mabie PC, Kessler JA. Development changes in progenitor cell responsiveness to cytokines. J Neurosci Res 1999;56:131-45.

(19) Kawaguchi A, Miyata T, Sawamoto K, Takashita N, Murayama A, Akamatsu W, et al. Nestin-EGFP transgenic mice: Viusualization of the self-renewal and multipotency of CNS stem cells. Mol Cell Neurosci 2001;17:259-73.

(20) Ramalho-Santos M, Yoon S, Matsuzaki Y, Mulligan RC, Melton DA. "Stemness": Transcriptional profiling of embryonic and adult stem cells. Science 2002;298:601-4.

(21) Ivanova NB, Dimos JT, Schaniel C, Hackney JA, Moore KA, Lemischka IR. A stem cell molecular signature. Science 2002;298:597-600.

(22) Terskikh AV, Easterday MC, Li L, Hood L, Kornblum HI, Geschwind DH, et al. From hematopoiesis to neuropoiesis: Evidence of overlapping genetic programs. Proc Natl Acad Sci 2001;98:7934-9.

(23) Molofsky A, Pardal R, Iwashita T, Park I, Clarke M, Morrison S. Bmi-1 dependence distinguishes neural stem cell self-renewal from progenitor proliferation. Nature 2003;425:962-7.

(24) Kelly S, Bliss T, Shah A, Sun G, Ma M, Foo W, et al. Transplanted human fetal neural stem cells survive, migrate, and differentiate in ischemic rat cerebral cortex. Proc Natl Acad Sci USA 2004;101:11839-44,.

(25) Burnstein R, Foltynie T, He X, Menon D, Svendsen C, Caldwell M. Differentiation and migration of long term expanded human neural progenitors in a partial lesion model of Parkinson's disease. Int J Biochem Cell Biol 2004;36:7023.

(26) McBride J, Behrstock S, Chen E, Jakel R, Siegel I, Svendsen $\mathrm{C}$, et al. Human neural stem cell transplants improve motor function in a rat model of Huntington's disease. J Comp Neurol 2004;475:211-9.
(27) Groszer M, Erickson R, Scripture-Adams DD, Lesche R, Trumpp A, Zack JA, et al. Negative regulation of neural stem/progenitor cell proliferation by the Pten tumor suppressor gene in vivo. Science 2001;294:2186-9.

(28) Grandbarbe L, Bouissac J, Rand M, Hrabé de Angelis M, Artavanis-Tsakonas S, Mohier E. Delta-Notch signaling controls the generation of neurons/glia from neural stem cells in a stepwise process. Development 2003;130:1391-402.

(29) Shimazaki T, Shingo T, Weiss S. The ciliary neurotrophic factor/leukemia inhibitory factor/gp130 receptor complex operates in the maintenance of mammalian forebrain neural stem cells. J Neurosci 2001;21:7642-53.

(30) Dontu G, Abdallah W, Foley J, Jackson K, Clarke M, Kawamura $\mathrm{M}$, et al. In vitro propagation and transcriptional profiling of human mammary stem/progenitor cells. Gene Dev 2003;17:1253-70.

(31) Scheffle B, Horn MIB, Laywell E, Coomes D, Kukekov V, et al. Marrow-mindedness: A perspective on neuropoiesis. Trends Neurosci 1999;22:348-57.

(32) Dontu G, Jackson K, McNicholas E, Kawamura M, Abdallah W, Wicha M. Role of Notch signaling in cell-fate determination of human mammary stem/progenitor cells. Breast Cancer Res 2004;6:R605-15.

(33) Bunting KD. ABC transporters as phenotypic markers and functional regulators of stem cells. Stem Cells 2002;20:11-20.

(34) Scharenberg CW, Harkey MA, Torok-Storb B. The ABCG2 tranporter is an efficient Hoechst 33342 efflux pump and is preferentially expressed by immature human hematopoietic progenitors. Blood 2002;99:507-12.

(35) Welm BE, Tepera SB, Venezia T, Graubert TA, Rosen JM, Goodell MA. Sca-1(pos) cells in the mouse mammary gland represent an enriched progenitor cell population. Dev Biol 2002;245:42-56.

(36) Stingl J, Eaves CJ, Zandieh I, Emerman JT. Characterization of bipotent mammary epithelial progenitor cells in normal adult human breast tissue. Breast Cancer Res Treat 2001;67:93-109.

(37) Stingl J, Eaves CJ, Kuusk U, Emerman JT. Phenotypic and functional characterization in vitro of a multipotent epithelial cell present in the normal adult human breast. Differentiation 1998;63:201-13.

(38) Kuperwasser C, Chavarria T, Wu M, Magrane G, Gray J, Carey L, et al. Reconstruction of functionally normal and malignant human breast tissues in mice. PNAS 2004;101:496671.

(39) Toma J, Akhavan M, Fernandes K, Barnabe-Heider F, Sadikot A, Kaplan D, et al. Isolation of multipotent adult stem cells from the dermis of mammalian skin. Nat Cell Biol 2001;3:778-84.

(40) Beltrami AP, Barlucchi L, Torella D, Baker M, Limana F, Chimenti S, et al. Adult Cardiac Stem Cells Are Multipotent and Support Myocardial Regeneration. Cell 2003;114:763-76.

(41) Li H, Liu H, Heller S. Pluripotent stem cells from the adult mouse inner ear. Nature medicine 2003;9:1293-9.

(42) Reya T, Morrison SJ, Clarke MF, Weissman IL. Stem cells, cancer, and cancer stem cells. Nature 2001;414:105-11.

(43) Bonnet D, Dick JA. Human acute myeloid leukemia is organized as a hierarchy that originates from a primitive hematopoietic cell. Nat Med 1997;3:730-7.

(44) Warner J, Wang J, Hope K, Jin L, Dick J. Concepts of human leukemic development. Oncogene 2004;23:23. 
(45) Al-Hajj M, Wicha M, Benito-Hernandez A, Morrison S, Clarke M. Prospective identification of tumorigenic breast cancer cells. Proc Nat Acad Sci USA 2003;100:3983-8.

(46) Singh SK, et al. Identification of a cancer stem cell in human brain tumors. Cancer Res 2003;63:5821-8.

(47) Singh S, Hawkins C, Clarke I, Squire J, Bayani J, Hide T. Henkelman R., Cusimano M, Dirks, P. Identification of human brain tumour initiating cells. Nature 2004;432:396401.

(48) Ignatova TN et al. Human cortical glial tumors contain neural stem-like cells expressing astroglial and neuronal markers in vitro. Glia 2002;39:193-206.

(49) Gabay L, Lowell S, Rubin L, Anderson D. Deregulation of dorsoventral patterning by FGF confers trilineage differentiation capacity on CNS stem cells in vitro. Neuron 2003;40:485-99.

(50) Angst B, Marcozzi C, Magee A. The cadherin superfamily: Diversity in form and function. J Cell Sci 2001;114:629-41.

(51) Vallorosi C, Day K, Zhao X, Rashid M, Rubin M, Johnson $\mathrm{K}$, et al. Truncation of the beta-catenin binding domain of E-cadherin precedes epithelial apoptosis during prostate and mammary involution. J Biol Chem 2000;275:3328-34.

(52) Meniel V, Clarke A. Wnt-Cadherin Connections in Normal and Neoplastic mammary Epithelium. J Mammary Gland Biol Neoplasia 2003;8:435-47.

(53) Arias AM, Brown AMC, Brennan K. Wnt signalling: pathway or network? Curr Opin Gene Dev 1999;9:447-54.

(54) Nusse R, Varmus HE. Wnt Genes. Cell 1992;69:1073-87.

(55) Brennan K, Brown AMC. Wnt Proteins in Mammary Development and Cancer. J Mammary Gland Biol Neoplasia 2003;9:119-31.

(56) Kielman M, Rindapaa M, Gaspar C, van Poppe LN, Breukel $\mathrm{C}$, van Leeuwen S, et al. Apc modulates embryonic stem-cell differentiation by controlling the dosage of beta-catenin signaling. Nat Gene 2002;32:594-605.

(57) Yamashita Y, Jones DL, Fuller MT. Orientation of asymmetric stem cell division by the APC tumor suppressor and centrosome. Science 2003;301:1547-50.

(58) Conacci-Sorrell M, Zhurinsky J, Ben-Ze'ev A. The cadherincatenin adhesion system in signaling and cancer. J Clin Invest 2002;109:987-91.

(59) Conacci-Sorrell M, Simcha I, Ben-Yedidia T, Blechman J, Savagner P, Ben-Ze'ev A. Autoregulation of E-cadherin expression by cadherin-cadherin interactions: the roles of betacatenin signaling, Slug, and MAPK. J Cell Biol 2003;163:84757.

(60) Boussadia O, Kutsch S, Hierholzer A, Delmas V, Kemler R. E-cadherin is a survival factor for the lactating mouse mammary gland. Mech Dev 2002;115:53-62.

(61) Jamora C, DasGupta R, Kocieniewski P, Fuchs E. Links between signal transduction, transcription and adhesion in epithelial bud development. Nature 2003;422:317-22.
(62) Smith GH, Chepko G. Mammary epithelial stem cells. Microsc Res Tech 2001;52:190-203.

(63) Trosko JT. He role of stem cells and gap junctional intercellular communication in carcinogenesis. J Biochem Mol Biol 2003;36:43-8.

(64) Fujita N, Jaye D, Kajita M, Geigerman C, Moreno C, Wade PA. MTA3, a Mi-2/NuRD complex subunit, regulates an invasive growth pathway in breast cancer. Cell 2003;113:20719.

(65) Brown AMC. Wnt signaling in breast cancer: Have we come full circle? Breast Cancer Res 2001;3:351-5.

(66) Brisken C, Heineman A, Chavarria T, Elenbaas B, Tan J, Dey S, et al. Essential function of Wnt-4 in mammary gland development downstream of progesterone signaling. Gene Dev 2000;14:650-4.

(67) Uyttendaele H, Soriano JV, Montesano R, Kitajewski J. Notch4 and Wnt-1 proteins function to regulate branching morphogenesis of mammary epithelial cells in an opposing fashion. Dev Biol 1998;196:204-17.

(68) Hennighausen L, Robinson GW. Signaling pathways in mammary gland development. Dev Cell 2001;1:467-75.

(69) Michaelson J, Leder P. Beta-catenin is a downstream effector of Wnt-mediated tumorigenesis in the mammary gland. Oncogene 2004;20:5093-9.

(70) Li Y, Welm B, Podsypanina K, Huang S, Chamorro M, Zhang $\mathrm{X}$, et al. Evidence that transgenes encoding components of the Wnt signaling pathway preferentially induce mammary cancers from progenitor cells. Proc Natl Acad Sci USA 2003;100:15853-8.

(71) Taipale J, Beachy PA. The hedgehog and Wnt signalling pathways in cancer. Nature 2001;411:349-54.

(72) Catriona HM, Jamieson C, Ailles L, Dylla S, Muijtjens M, Jones C, Zehnder J, et al. Granulocyte-Macrophage progenitors as candidate leukemic stem cells in blast-crisis CML. New Engl J Med 2004;351:657-67.

(73) Reya T, Duncan A, Ailles L, Domen J, Scherer D, Willert K, et al. A role for Wnt signalling in self-renewal of haematopoietic stem cells. Nature 2003;423:409-14.

(74) Kuhnert F, Davis C, Wang H, Chu P, Lee M, Yuan J, et al. Essential requirement for Wnt signaling in proliferation of adult small intestine and colon revealed by adenoviral expression of Dickkopf-1. Proc Natl Acad Sci USA 2004;101:266-71.

(75) Brittan M, Wright N. Gastrointestinal stem cells. J Pathol 2002;197:492-509.

(76) Zhu A, Watt F. Beta-catenin signalling modulates proliferative potential of human epidermal keratinocytes independently of intercellular adhesion. Development 1999;126:126(10):2285-98, 1999.

(77) Liu B, McDermott S, Khwaja S, Alexander C. The transforming activity of Wnt effectors correlates with their ability to induce the accumulation of mammary progenitor cells. Proc Natl Acad Sci USA 2004;101:4158-63. 